This is a paper submitted to and accepted for publication in:

Mu-Chieh Lo, Robinson Guzmán, Carlos Gordón and Guillermo Carpintero. "Mode-locked laser with pulse interleavers in a monolithic photonic integrated circuit for millimeter wave and terahertz carrier generation". Opt. Lett. 42 (8), 1532-1535 (2017).

https://doi.org/10.1364/OL.42.001532

(C) 2017 Optical Society of America 


\title{
Mode-locked laser with pulse interleavers in monolithic photonic integrated circuit for millimeter wave and terahertz carrier generation
}

\author{
Mu-Chieh Lo, ${ }^{1,}{ }^{*}$ Robinson Guzmán, ${ }^{1}$ CARlos Gordón, ${ }^{2}$ And Guillermo \\ CARPINTERO ${ }^{1}$ \\ ${ }^{1}$ Departamento de Tecnología Electrónica, Universidad Carlos III de Madrid, Av de la Universidad, 30. 28911 Leganés, Madrid, Spain \\ ${ }^{2}$ Facultad de Ingeniería en Sistemas, Electrónica e Industrial. Universidad Técnica de Ambato, 180150 Ambato, Ecuador \\ *Corresponding author: mlo@ing.uc3m.es
}

Received 20 December 2016; revised 8 March 2017; accepted 19 March 2017; posted 20 March 2017 (Doc. ID 282898); published 6 April 2017

This paper presents a photonics-based mmW and $\mathrm{THz}$ frequency synthesizer using monolithic InP photonic integrated circuit comprising a mode-locked laser (MLL) and two pulse interleaver stages to multiply the repetition rate frequency. The MLL is a multiple colliding pulse mode-locked laser producing an $80 \mathrm{GHz}$ repetition rate pulse train. Through two consecutive monolithic pulse interleaver structures, each doubling the repetition rate, we demonstrate the achievement of $160 \mathrm{GHz}$ and $320 \mathrm{GHz}$. The fabrication was done on a multi-project wafer (MPW) run of a generic InP photonic technology platform.

OCIS codes: (140.4050) Mode-locked lasers; (140.5960) Semiconductor lasers; (250.5300) Photonic integrated circuits.

https://doi.org/10.1364/OL.42.001532

The continuous increase in the memory size on mobile devices and the increasing resolution of new TV standards $4 \mathrm{~K}$ and $8 \mathrm{~K}$ are demanding higher wireless data rates. Also, the next $5 \mathrm{G}$ generation wireless systems and the telecommunications standards beyond the current 4G/IMT-Advanced are expected to increase the required bandwidth, reaching $100 \mathrm{Gbit} / \mathrm{s}$ by 2020 [1]. For such ultra-high speed wireless links, researchers have been attempting to exploit the higher end of the millimeter wave range (mmW, 30 $\mathrm{GHz}$ to $300 \mathrm{GHz}$ ) and the Terahertz (THz, $300 \mathrm{GHz}$ to $3 \mathrm{THz}$ ) region of the frequency spectrum [2]. The lack of suitable sources has maintained these regions unused, coining the term of THz gap [3]. To fill this gap, photonic techniques have been considered for high frequency generation, mainly through optical heterodyning and pulsed techniques.

Pulsed sources are usually based on mode-locked laser (MLL) structures. MLLs optical spectrum is characterized by phase locked longitudinal modes which lead to a train of optical pulses in the time domain at a repetition frequency defined by the cavity lengths and number of pulses in the cavity. For mmW and THz generation, MLL have been shown to generate more emitted power than heterodyning schemes [4]. MLLs in the form of photonic integrated circuits (PIC) are highly desirable to realize compact devices owing to the small footprint, lower power consumption, improved alignment and immunity to vibration [5].
One of the challenges of increasing the repetition rate is that the fundamental repetition rate frequency of MLL is inversely proportional to the cavity length. As repetition frequencies increase beyond $100 \mathrm{GHz}$, the short cavity that is required is not able to provide enough optical amplification to overcome the cavity loss. For this reason, schemes to raise the repetition rate without sacrificing the feasibility to accommodate gain sections must be addressed.

On-chip multiple colliding pulse mode-locked laser (mCPML) is a promising solution to this problem [6-12]. In mCPML configuration, multiple saturable absorbers are located at precise locations which divide the cavity into sub-sections with integer length ratios. This causes the cavity to support multiple optical pulses, thus increasing the repetition rate to a multiple of the fundamental frequency defined by the cavity length. Repetition rates at $60 \mathrm{GHz}$ [6], $100 \mathrm{GHz}$ [7], $192 \mathrm{GHz}$ [8], $237 \mathrm{GHz}$ [9], $240 \mathrm{GHz}$ [10], $375 \mathrm{GHz}$ [11], $377 \mathrm{GHz}$ [12], and $860 \mathrm{GHz}$ [13] were realized. These demonstrations used cleaved facets as reflectors to define the cavity [6,8-13], which is not an accurate process, causing uncertainty in the cavity length. Recently, on-chip Multimode Interference Reflectors (MIR) [14] were demonstrated [7] to provide more accurate design.

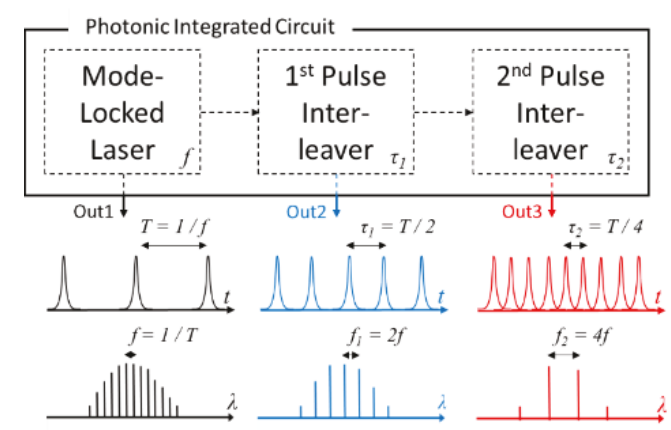

Fig. 1. Block diagram of the proposed PIC, based on a MLL with repetition rate $f$ followed by two pulse interleaver stages, with delays $\boldsymbol{\tau}_{1}$ and $\boldsymbol{\tau}_{2}$, respectively. 

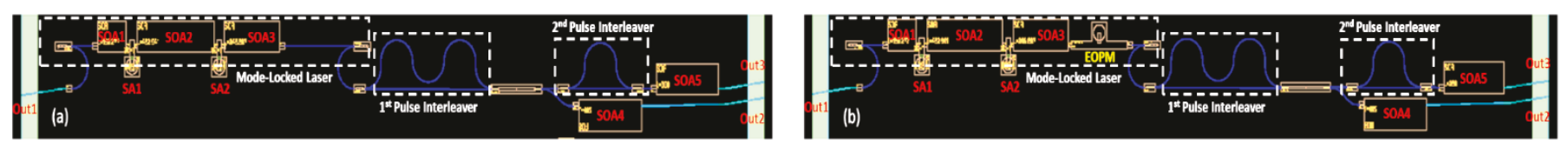

Fig. 2. Schematic layout of the two designs, PIC-a (left), without EOPM, and PIC-b (right), with EOPM. EOPM: electro-optic phase modulator.

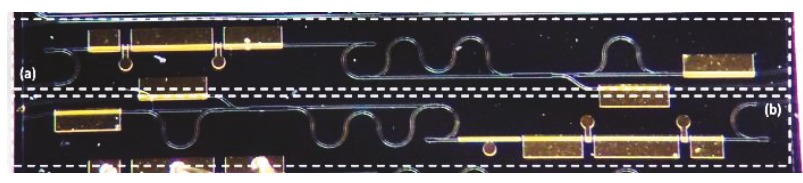

Fig. 3. Microscope image of PIC-a (top) and PIC-b (bottom), with EOPM.

The advantage of monolithic integration of on-chip mCPML can be further exploited to increase the pulse train by optical processing on the same chip. Optical multiplication to increase the repetition rate can be achieved through interleaver stages based on asymmetric Mach-Zehnder interferometer (MZI), introducing an optical delay on one of the arms of half the repetition period, at the cost of $3 \mathrm{~dB}$ optical loss [15]. The interleaving in the time domain is accompanied by the filtering effect in the frequency domain. To realize such a pulse interleaver the MLL optical frequency comb lines should be aligned to the passband of MZI filter, implying that including additional spectral tuning mechanism is favorable.

In this paper, we present a novel type of MLL structure, with the block diagram shown in Fig. 1, combining an on-chip mCPML with two MZI pulse interleaver stages. The structure exhibits three output ports, Out1 with the mCMLL repetition rate, and Out2 and Out3 from each interleaver stage, each doubling the input repetition rate. This is the first time that the carrier generator structure is monolithically integrated pushing the frequency up to mmW and THz. To reach $300 \mathrm{GHz}$, currently being proposed for transmission systems enabling $100 \mathrm{~Gb} / \mathrm{s}$ data rates [16], we designed our MLL with an $80 \mathrm{GHz}$ repetition rate (Period between optical pulses $\boldsymbol{T}=1 / \boldsymbol{f}=12.50 \mathrm{ps}$ ). The MLL is followed by two interleaver stages. The first stage has a delay $\boldsymbol{\tau}_{\boldsymbol{1}}=\boldsymbol{T} / 2=6.25 \mathrm{ps}$ and the second $\boldsymbol{\tau}_{2}=\boldsymbol{T} / 4=3.13 \mathrm{ps}$. For this approach, we used the same library of standardized building blocks (BBs) and commercial multi-project wafer (MPW) foundry SMART Photonics InP active/passive integration service Error! Reference source not found. that we used to demonstrate the MCPML structure.

The schematic layout of the two designs (PIC-a and PIC-b) included in the chip are shown in Fig. 2, while the microscope photograph of the fabricated samples is shown in Fig. 3. On both, the core component is a mCPML, including two pulse interleavers. All the optical output waveguides are terminated in $7^{\circ}$ angled shallowly etched waveguides to avoid back reflections. On the lefthand side, Out1 outputs the mCPML pulse train, with period $\boldsymbol{T}$. On the right-hand side, Out 2 and Out 3 output the $\mathrm{x} 2$ and $\mathrm{x} 4$ multiplied pulse trains, respectively. The difference between the two designs is the intracavity electro-optic phase modulator (EOPM) for spectral tuning in Fig. 2(b). The area containing these two PICs is $4.7 \mathrm{~mm}$ long and $0.8 \mathrm{~mm}$ wide. Shallowly etched waveguides are $2 \mathrm{um}$ wide and deeply etched waveguides are $1.5 \mu \mathrm{m}$ wide, with propagation losses are $2-3 \mathrm{~dB} / \mathrm{cm}$, resulting in propagation losses of $2 \mathrm{~dB}$ for the longest path in the two stages of interleaver. Each interleaver stage introduces $6 \mathrm{~dB}$ loss, $3 \mathrm{~dB}$ due to power splitting at the multimode interference (MMI) divider and another $3 \mathrm{~dB}$ at the MMI combiner, resulting in a $12 \mathrm{~dB}$ total loss for the two stages of interleaver [19]. This is not a major issue, since our approach using monolithic integration enables us to add SOAs through the design, i.e. prior to each pulse interleaver stage.

Mode-Locked Laser: Since there is a minimum gain required to overcome the cavity loss, to realize the $80 \mathrm{GHz}$ repetition rate we use a mCPML with a cavity length of $2 \mathrm{~mm}$, to which corresponds a fundamental repetition rate of $20 \mathrm{GHz}$. This cavity is terminated by a pair of 2-port multimode interference reflectors (MIRs), with 50\% reflectivity, defining a Fabry-Pérot resonator. Two saturable absorber (SA) sections are precisely located within the resonator, one at the midpoint SA2 $(30 \mu \mathrm{m})$, and the other, SA1 $(30 \mu \mathrm{m})$ at $1 / 4$ total cavity length from the left MIR end. This arrangement quadruples the frequency, increasing the repetition rate from the fundamental round trip frequency $20 \mathrm{GHz}$ to $80 \mathrm{GHz}$.

The two SA sections within the resonator define three gain sections, each having a semiconductor optical amplifier with different length, SOA1 (180 $\mu \mathrm{m})$, SOA2 (480 $\mu \mathrm{m})$ and SOA3 (350 $\mu \mathrm{m})$. In PIC-b, an electro-optic phase modulator (EOPM: $320 \mu \mathrm{m}$ ) is inserted beside SOA3 for spectral fine tuning as shown. All these active sections are all based on the same InGaAsP multi-quantum well core. The active-passive integration technology enables the use of passive waveguides to have the appropriate length relationship between amplification and absorption. Besides, isolation sections are inserted between adjacent active components to prevent unwanted current flow. Active and passive waveguides are jointed via transition sections. The MLL has optical outputs at each MIR mirror. From the left end MIR, we provide a direct access to the MLL pulse train at Out1. From the right end of the MLL output, the emitted pulse train goes to further on-chip optical signal processing into the pulse interleaver stages.

$1^{\text {st }}$ Pulse Interleaver and $2^{\text {nd }}$ Pulse Interleaver: To increase the repetition rate by a factor of two and four times, we use two OTDM-based consecutive pulse interleaver stages. The pulse interleavers are asymmetric MZIs, in which optical delay line corresponding to half and quarter of pulse train's period respectively. The delay line arrangement is realized by using straight and curved passive waveguides providing length control with lithographic accuracy.

The first pulse interleaver stage, the upper arm of the MZI is adjusted to $1.3 \mathrm{~mm}$, while the lower arm is a straight waveguide of $0.8 \mathrm{~mm}$. This difference in path lengths introduces optical delay of $0.50 \mathrm{~mm}$ in the upper arm, corresponding to a time delay of $\boldsymbol{\tau}_{\mathbf{1}}$, so the pulses traveling through this upper arm are delayed half of the repetition rate period, being interleaved at the MMI combiner. Similarly, in the second pulse interleaver the upper arm is a combination of curved waveguide of $0.65 \mathrm{~mm}$ in total and the lower branch is $0.40 \mathrm{~mm}$ long. In this stage, the path length difference is $0.25 \mathrm{~mm}$, so that $\boldsymbol{\tau}_{2}$ is a quarter of repetition period.

We have access to the signal of each interleaver stage, which are connected to output ports. For the first pulse interleaver, the output waveguide includes a booster amplifier SOA4 $(400 \mu \mathrm{m})$ prior to Out2. The pulse train that travels through the cascade of the two pulse interleavers is connected to Out3 through another booster amplifier SOA5 $(400 \mu \mathrm{m})$. Consequently, at Out2 and Out3 the frequency doubling and quadrupling can be observed. 

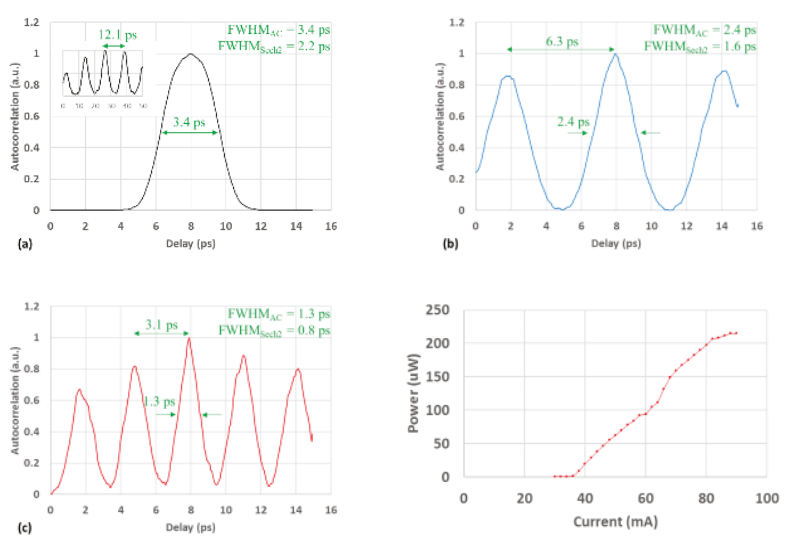

Fig. 4. PIC-a experimental results: (a) Autocorrelation at Out1, (b) Autocorrelation at Out2 (1st Interleaver), (c) Autocorrelation at Out3 (2nd Interleaver) and, (d) LightCurrent characteristic at Out1

To demonstrate the optical pulse multiplication, we have measured the resulting pulse train at each of the chip outputs with a second harmonic generation autocorrelation using an APE pulse check autocorrelator. The results of the signal at Out1-3 of PIC-a with span of 15 ps are shown in Fig. 4. In Fig. 4(a), for the MLL output, the full width at half maximum (FWHM) is 3.4 ps. Assuming a hyperbolic secant pulse shape, the pulse-width is $2.2 \mathrm{ps}$. The biasing condition is as follows: SOA1: 5 mA, SOA2+SOA3: $81 \mathrm{~mA}$, SA1: $-0.8 \mathrm{~V}$, SA2: $-0.8 \mathrm{~V}$. From an autocorrelation trace with $50 \mathrm{ps}$ span, we measured a time separation between adjacent pulses of 12.1 ps, equivalent to a repetition rate of $82 \mathrm{GHz}$. The autocorrelation traces of the doubled pulse train from Out2 is shown in Fig. 4(b). In this output, the measured FWHM is 2.4 ps, with corresponding pulse-width of $1.6 \mathrm{ps}$. In this case, the spacing between adjacent pulses is $6.3 \mathrm{ps}$, corresponding to $159 \mathrm{GHz}$ repetition rate. The bias conditions were set to SOA1: $9 \mathrm{~mA}$, SOA2+SOA3: $88 \mathrm{~mA}$, SA1: -0.7 V, SA2: -0.6 V, SOA4: $30 \mathrm{~mA}$ to optimize the pulse signal at this output. Finally, Fig. 4(c) presents the autocorrelation trace at Out3, where we measured a 1.3 ps FWHM, corresponding to a 0.8 ps pulse-width. The measured repetition rate is is $323 \mathrm{GHz}$, from the 3.1 ps time spacing. The condition were SOA1: 9 mA, SOA2+SOA3: 83 mA, SA1: -0.7 V, SA2: -1.0 V, EOPM: 1.0 V, SOA5: $34 \mathrm{~mA}$. We have observed that the pulse width reduces as the pulses cross through the optical pulse interleaving stages doubling the repetition rate. The origin of this phenomenon is now under investigation. We optimized the biasing conditions when taking the measurements from each output, which in turn affects the pulse shape. This tuning was required due to the lack of phase tuning components in the arms of the interleaver arms to adjust the time delays, which left us only with the MLL bias parameters for adjustment.

To assess the optical power, we measured the Light-Current characteristic, shown in Fig. 4(d). The device has a threshold current around $36 \mathrm{~mA}$. From this curve, we also observe that the maximum optical output power from Out1, coupled to a lensed fiber, was 200 $\mu \mathrm{W}$ when ISOA2+SOA3 $=80 \mathrm{~mA}$. The rest of bias conditions were SOA1: $9 \mathrm{~mA}$, SA1: $0 \mathrm{~V}, \mathbf{S A 2}$ : $0 \mathrm{~V}$. This is also the power level that we observed from the other outputs (Out2 and 3), demonstrating that the booster SOAs allow us to recover the pulse interleaver losses. This points to the fact that this chip can be used as a carrier wave source for $\mathrm{THz}$ wireless links, if additional SOA amplifiers are inserted in the design prior to each interleaver stage as previously discussed. We have already demonstrated that mCPML structures, including a booster SOA, can produce up to $0 \mathrm{dBm}$ optical power coupled into the fiber [7]. The generic technology platform that we used offers $70 \mathrm{~cm}^{-1}$ gain material, and thus a $197 \mu \mathrm{m}$ long SOA would compensate for the $6 \mathrm{~dB}$ interleaver loss. This would enable us to reach the $0 \mathrm{dBm}$ optical source output level required from a THz wireless transmission link budget calculation [2].

In Fig. 5 the optical spectrum at Out1-3 measured on Yokogawa AQ6370B optical spectrum analyzer (OSA) with $0.02 \mathrm{~nm}$ resolution bandwidth (res) are shown within 1545 - 1555 nm. In Fig. 5(a) the spectrum collected at Out1 resembles a typical Gaussian-like optical frequency comb generated by MLL where the 7 8 comb lines are evenly distributed and has $-13 \mathrm{dBm} / \mathrm{res}$ at highest level. The comb lines stand side by side with the same FSR of $0.65 \mathrm{~nm}$ corresponding to $81 \mathrm{GHz}$. Aside from these dominant comb line modes, there are other lower modes whose power is around -50 $\mathrm{dBm} / \mathrm{res}$. The inset presents the detail around $1549 \mathrm{~nm}$ where the suppressed lines have $0.16 \mathrm{~nm}(20 \mathrm{GHz})$ spacing. Three modes are suppressed within every four modes. This agrees with the design of mCPML in which fourth harmonic components are obtained. The side-mode suppression ratio (SMSR) is larger than $30 \mathrm{~dB}$. These measurements were taken prior to the autocorrelation traces, under a different set of bias conditions. From these measurements, we have identified that autocorrelation traces are key to optimize the interleaver performance, which require fine tuning of the repetition rate to match the designed delays.
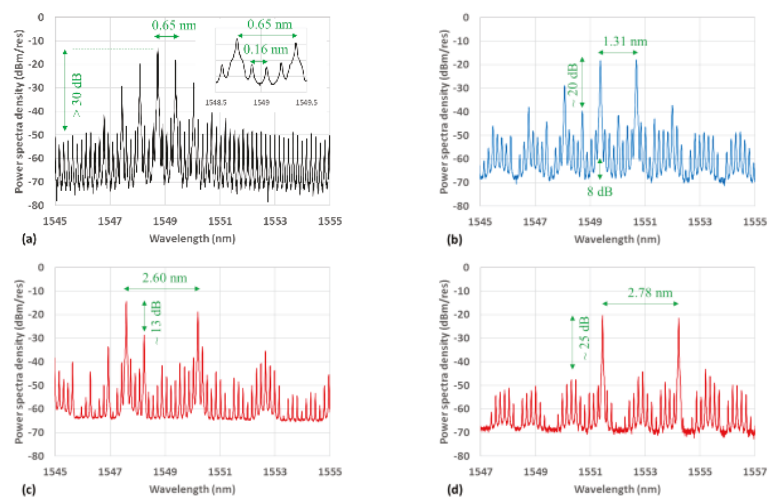

Fig. 5. Optical spectrum (a-c) PIC-a (d) PIC-b (with EOPM)

(a)Out1(Mode-Locked): FSR $0.65 \mathrm{~nm}$; SMSR: $30 \mathrm{~dB}$

(b)Out2(1st Interleaver): FSR $1.31 \mathrm{~nm}$; SMSR: $20 \mathrm{~dB}$

(c)Out3(2nd Interleaver): FSR $2.60 \mathrm{~nm}$; SMSR: $13 \mathrm{~dB}$

(d)Out3(2nd Interleaver): FSR: $2.78 \mathrm{~nm}$; SMSR: $25 \mathrm{~dB}$

The quality of the electrical signal that is generated from mCPML structures in the passive mode locking regime has been already presented, comparing the electrical linewidth of devices with repetition rate frequencies falling within the range of our electrical spectrum analyzer (up to $40 \mathrm{GHz}$ ) with devices requiring an external mixer head [20]. Previous results show that the electrical linewidth from the core MLL is within the MHz range, as is usual for passive mode locked semiconductor lasers operating at the fundamental frequency. Furthermore, we expect that under hybrid mode locking, the increased stability of the MLL pulse train will also have an impact on the multiplied versions generated from the interleavers. Further studies are necessary to establish the phase noise dependence on the number of interleaver stages.

Fig. 5(b) is the optical spectrum collected at Out2. Like Fig. 5(a), it shows an asymmetric frequency comb with $-18 \mathrm{dBm} / \mathrm{res}$ at peak 
and wider spacing of $1.31 \mathrm{~nm}$, which is double the spacing in Fig. 5(a) and corresponds to about $164 \mathrm{GHz}$. Compared to Fig. 5(a), every other comb line is suppressed. The SMSR is roughly $20 \mathrm{~dB}$. The wavy baseline of the optical spectrum is caused by the MZI filtering behavior which is absent in Fig. 5(a). We realized that this filtering effect can be used to enhance the SMSR if modes of interest are situated in the passband. In this case this effect improves SMSR by around $5 \mathrm{~dB}$. The spacing of lower modes is still $0.16 \mathrm{~nm}$, same as in Fig. 5(a).

The spectrum at Out3 is presented in Fig. 5(c), in which the two interleaver stages result in a frequency quadrupling. The spectral spacing between dominant modes is $2.6 \mathrm{~nm}$, four times the spacing in Fig. 5(a). However, the comb-like spectrum does not appear as clear as the previous two. Few modes are not suppressed as expected which distort the comb. Also, the two dominant modes are not at the same power level. It is believable that this distortion results from the mismatch between MLL repetition rate frequency and the delay in MZI, as well as the imperfect spectral relationship between MLL comb and MZI filter.

To fine tune for the mismatch, the EOPM in PIC-b was fed with controllable voltage. The optical spectrum at Out3 of PIC-b is shown in Fig. 5(d). (SOA1: $10 \mathrm{~mA}$, SOA2+SOA3: $75 \mathrm{~mA}$, SA1: -0.7 V, SA2: -0.7 V, EOPM: -2.0 V, SOA5: $34 \mathrm{~mA}$ ). The wavelength spacing is $2.78 \mathrm{~nm}(350 \mathrm{GHz})$, even greater than expected for which further investigation is required. The mode spacing between lower lasing modes is still $0.16 \mathrm{~nm}$, same as in Fig. 5. It has a greater SMSR of around $25 \mathrm{~dB}$. The PIC-b (with EOPM) has one more degree of freedom that is the voltage for EOPM enabling a tuning mechanism. However, the tuning capability is limited as phase tuning conflicts with multiple colliding pulse mode locking. The induced phase shift changes the optical path length, leading to a de-tuning from the certain length ratio of sub-cavities 1:1:2. Symmetric colliding pulse configuration with symmetric intra-cavity EOPM pair potentially provides wider tuning range without breaking the certain length ratio $1: 1$ [21].

We have developed a mmW and THz carrier generator PIC-a that emits $80 \mathrm{GHz}, 160 \mathrm{GHz}$, and $320 \mathrm{GHz}$ pulse train. This PIC comprises an $80 \mathrm{GHz}$ mCPML with $20 \mathrm{GHz}$ fundamental round trip frequency, and two optical delay line-assisted MZI interleavers as frequency multiplier. In the other similar design PIC-b, an additional EOPM is placed in the laser cavity for fine spectral tuning. By presenting the autocorrelation traces and optical spectra of this carrier generator its capability to produce $\mathrm{mmW}$ and THz signals is confirmed. It is estimated that the pulsewidth at $80 \mathrm{GHz}, 160 \mathrm{GHz}$ and $320 \mathrm{GHz}$ is $2.2 \mathrm{ps}, 1.6 \mathrm{ps}$ and $0.8 \mathrm{ps}$ and the SMSR is $30 \mathrm{~dB}, 20 \mathrm{~dB}$ and $13 \mathrm{~dB}$, respectively. The device PIC-b features spectral tuning by inserting a phase modulator. With EOPM-based tuning, the spectrum of PICb at Out3 exhibits a greater SMSR of $25 \mathrm{~dB}$ and a wider FSR of 350 $\mathrm{GHz}$.

Funding. European Union's Horizon 2020 research and innovation programme under the Marie Sklodowska-Curie grant agreement No. 642355; Spanish Ministerio de Economia y Competitividad DiDACTIC project (TEC2013-47753-C3-3-R).

Acknowledgment. We thank TU/e COBRA research institute and SMART Photonics for advanced building blocks and fabrication support.

\section{References}

1. T. Nagatsuma, G Carpintero "Recent Progress and Future Prospect of Photonics-Enabled Terahertz Communications Research" IEICE Trans. On Electronics E98-C(12) pp. 1060-1070 (2015)

2. A.J. Seeds, H. Shams, M.J. Fice, C.C. Renaud, "TeraHertz Photonics for Wireless Communications" J. of Lightwave Tech., 33(3) pp. 579 (2015)

3. S. Koenig, D. Lopez-Diaz, J. Antes, F. Boes, R. Henneberger, A. Leuther, A. Tessmann, R. Schmogrow, D. Hillerkuss, R. Palmer, T. Zwick, C. Koos, W. Freude, O. Ambacher, J. Leuthold, and I. Kallfass "Wireless sub-THz communication system with high data rate." Nature Photonics 7, no. 12 (2013): 977-981.

4. C. Renaud, A. Stöhr, T. Goebel, F. Van Dijk, and G. Carpintero. "Selected Photonic THz Technologies." Semiconductor TeraHertz Technology: Devices and Systems at Room Temperature Operation (2015): 304.

5. Coldren, Larry A., Scott W. Corzine, and Milan L. Mashanovitch. Diode lasers and photonic integrated circuits. Vol. 218. John Wiley \& Sons, 2012.

6. Y. Li, F. L. Chiragh, Y. Xin, C. Lin, J. Kim, C. G. Christodoulou, and L. F. Lester, "Harmonic mode-locking using the double interval technique in quantum dot lasers," Opt. Express 18, 14637-14643 (2010)

7. C. Gordón, R. Guzmán, V. Corral, M-C. Lo and G. Carpintero, "On-Chip Multiple Colliding Pulse Mode-Locked Semiconductor Laser," in Journal of Lightwave Technology, vol. 34, no. 20, pp. 4722-4728, Oct.15, 152016

8. Y. Katagiri and A. Takada, "A harmonic colliding-pulse mode-locked semiconductor laser for stable subterahertz pulse generation," in IEEE Photonics Technology Letters, vol. 9, no. 11, pp. 1442-1444, Nov. 1997.

9. A. R. Rae, M. G. Thompson, R. V. Penty, I. H. White, A. R. Kovsh, S. S. Mikhrin, D. A. Livshits, and I. L. Krestnikov, "Harmonic mode-locking of a quantumdot laser diode," in Proc. 19th Annu. Meeting IEEE, Lasers Electro-Opt. Soc., Montreal, Cabada, Oct./Nov. 2006, pp. 874-875.

10. L. Hou, M. Haji, and J. H. Marsh, "240 GHz pedestal-free collidingpulse mode-locked laser with a wide operation range," Laser Phys. Lett. , vol. 11, no. 11, pp. 115804, 2014

11. J. F. Martins-Filho, E. Avrutin, C. N. Ironside, and J. S. Roberts, "Monolithic multiple colliding pulse mode-locked quantum-well lasers, experiment and theory," IEEE J. Sel.Top. Quantum Electron., vol. 1, no. 2, pp. 539-551, June 1995.

12. T. Shimizu, X. L. Wang, and H. Yokoyama, "Asymmetric colliding-pulse mode-locking in InGaAsP semiconductor lasers," Opt. Rev. 2, 401-403 (1995).

13. T. Shimizu, I. Ogura and H. Yokoyama, " $860 \mathrm{GHz}$ rate asymmetric colliding pulse modelocked diode lasers," in Electronics Letters, vol. 33, no. 22, pp. 1868-1869, 23 Oct 1997.

14. E. Kleijn, M. K. Smit and X. J. M. Leijtens, "Multimode Interference Reflectors: A New Class of Components for Photonic Integrated Circuits," in Journal of Lightwave Technology, vol. 31, no. 18, pp. 3055-3063, Sept.15, 2013.

15. M.Y. Sander, S. Frolov, J. Shmulovich, E.P. Ippen, and F.X. Kärtner, "10 GHz femtosecond pulse interleaver in planar waveguide technology," Opt. Express 20, 4102-4113 (2012)

16. T. Nagatsuma, G. Ducournau, and C. Renaud. "Advances in terahertz communications accelerated by photonics." Nature Photonics 10, no. 6 (2016): 371-379.

17. M. Smit, X. Leijtens, E. Bente, J. Van der Tol, H. Ambrosius, D. Robbins, M. Wale, N. Grote, and M. Schell, IET Optoelectron. 5, 187 (2011).

18. SMART Photonics B.V. www.smartphotonics.nl

19. Joint European Platform for Photonic Integration of Components and Circuits. http://www.jeppix.eu/

20. C. Gordón, R. Guzmán, V. Corral, X. Leijtens, and G. Carpintero, "On-Chip Colliding Pulse Mode-locked laser diode (OCCP-MLLD) using multimode interference reflectors," Opt. Express 23, 14666-14676 (2015)

21. M.-C. Lo, R. Guzmán, C. Gordón, G. Carpintero , "Tunable Mode-Locked Laser Photonic Integrated Circuit Using Intracavity Phase Modulators" Optical Fiber Communications Conference and Exhibition (OFC) 2017, pp. W2A.1 